Check for updates

Cite this: Photochem. Photobiol. Sci., 2020, 19, 308

Received 20th November 2019, Accepted 23rd January 2020

DOI: 10.1039/c9pp00453j

rsc.li/pps

\section{High aldehyde dehydrogenase activity does not protect colon cancer cells against $\mathrm{TPCS}_{2 \mathrm{a}}$-sensi- tized photokilling}

\author{
Judith Jing Wen Wong (iD) and Pål Kristian Selbo (iD *
}

\begin{abstract}
Aldehyde dehydrogenases (ALDH) are detoxifying enzymes that are upregulated in cancer stem cells (CSCs) and may cause chemoand ionizing radiation (IR) therapy resistance. By using the ALDEFLUOR assay, CD133 + human colon cancer cells HT-29, were FACSorted into three populations: ALDH ${ }^{\text {bright }}, \mathrm{ALDH}^{\text {dim }}$ and unsorted (bulk) and treated with chemo-, radio- or photodynamic therapy (PDT) using the clinical relevant photosensitizer disulfonated tetraphenyl chlorin $\left(\mathrm{TPCS}_{2 a}\right.$ /fimaporfin). Here we show that there is no difference in cytotoxic responses to TPCS $_{2 a}-P D T$ in ALHD ${ }^{\text {bright }}, \mathrm{ALDH}^{\text {dim }}$ or bulk cancer cells. Likewise, both 5-FU and oxaliplatin chemotherapy efficacy was not reduced in ALDH ${ }^{\text {bright }}$ as compared to ALDH ${ }^{\text {dim }}$ cancer cells. However, we found that ALHD ${ }^{\text {bright }}$ HT-29 cells are significantly less sensitive to ionizing radiation compared to $\mathrm{ALDH}{ }^{\text {dim }}$ cells. This study demonstrates that the cytotoxic response to PDT (using TPCS $_{2 a}$ as photosensitizer) is independent of ALDH activity in HT-29 cancer cells. Our results further strengthen the use of $\operatorname{TPCS}_{2 a}$ to target CSCs.
\end{abstract}

Aldehyde dehydrogenases (ALDHs) constitute a group of enzymes that have been associated with cancer progression and cancer therapy resistance. ${ }^{1}$ ALDHs have diverse cellular activity, including vital role in detoxification of aldehydes to carboxylic acids, thereby preventing generation of reactive oxygen species (ROS) and lipid peroxidation. ${ }^{1}$ In addition, ALDHs are involved in the synthesis of retinoic acid, which is important for cell survival, proliferation, embryogenesis and development of the immune system. ${ }^{1}$ Overexpression of ALDH1 is used as a marker for both normal stem and progenitor cells and cancer stem cells (CSCs). ${ }^{2,3}$ High ALDH1 activity provides a survival advantage of CSC as they are more equipped to resist accumulation of toxic aldehydes induced by increased metabolic activity, ionizing radiation or ROS-generating drugs. ${ }^{1,4}$ In this communication, we present results obtained in fluorescence-activated cell sorted (BD FACS Aria II

Department of Radiation Biology, Institute for Cancer Research, The Norwegian Radium Hospital, Oslo University Hospital Montebello, 0379 Oslo, Norway. E-mail: Selbo@rr-research.no; Tel: (+47)22781469 cell sorter from Becton Dickinson (BD Biosciences, San Jose, USA)) human colon cancer cells with high (ALDH ${ }^{\text {bright }}$ ) and low $\left(\mathrm{ALDH}^{\mathrm{dim}}\right)$ ALDH activity. We compared these populations with regard to cytotoxic responses to chemotherapy, ionizing radiation or photodynamic therapy (PDT). For PDT, we selected the photosensitizer disulfonated tetraphenyl chlorin $\left(\mathrm{TPCS}_{2 \mathrm{a}} /\right.$ fimaporfin, PCI Biotech AS, Oslo, Norway) as TPCS $_{2 \mathrm{a}}$ is a clinical relevant photosensitizer used in the drug delivery technology photochemical internalization (PCI). ${ }^{5,6}$

The ALDEFLUOR assay (STEMCELL Technologies, Vancouver, Canada) was performed to evaluate ALDH activity and cell sorting. The assay is based on the use of BODIPY-aminoacetaldehyde (BAAA) which is a substrate of ALDH which convert BAAA into BODIPY-aminoacetate $\left(\mathrm{BAA}^{-}\right)$that is highly fluorescent and retained in live cells due to its negative charge. ${ }^{7}$ Thus, cells with high and low ALDH activity can be distinguished and sorted using flow cytometry based on the fluorescent signal from BAA ${ }^{-}$. The ALDH inhibitor, $N, N$-diethylaminobenzaldehyde (DEAB), was included as a control providing adequate gating strategy for flow cytometry. By flow cytometry (BD LSR II, BD Biosciences), we screened a panel of eight cancer cell lines for ALDH activity which included; HT-29 (human colorectal adenocarcinoma, ATCC ${ }^{\circ} H T B-38^{\mathrm{TM}}$ ), 5-FU-resistant and sensitive Panc 03.27derived monoclonal cell lines (human pancreatic adenocarcinoma generated as previously described, ${ }^{8}$ provided by Dr. Stephan Krauss), CT26.WT (murine undifferentiated colon carcinoma, ATCC ${ }^{2} C R L-2638^{\mathrm{TM}}$ ) and $4 \mathrm{~T} 1$ (murine triple negative mammary carcinoma, ATCC®CRL-2539 ${ }^{\mathrm{TM}}$ ) (Fig. 1).

A shift in fluorescence was observed in both murine cell lines, CT26.WT and 4T1, and in three of the 5-FU-resistant Panc 03.27-derived cell lines, Panc 03.27R-B1L, Panc 03.27R-B1Q and Panc 03.27R-B1LV, indicating homogenous ALDH activity. Interestingly, the 5-FU-sensitive Panc 03.27S-Nt and Pan03.27S-Nw cell lines, displayed heterogeneous ALDH activity compared to Panc 03.27R-B1L, -B1Q and B1V.

We have previously shown that the 5-FU-resistant Panc 03.27R-B1L, -B1Q and -B1V are hypersensitive to TPCS $_{2 \mathrm{a}}$-PDT compared to the 5 -FU sensitive clones. ${ }^{9}$ Based on this, and the 

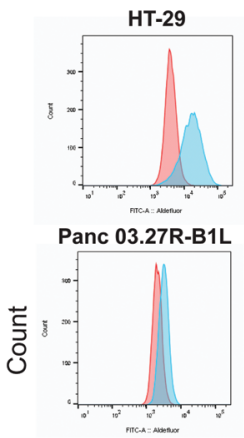

$4 \mathrm{T1}$

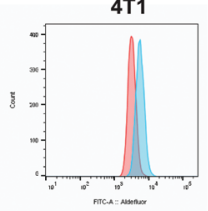

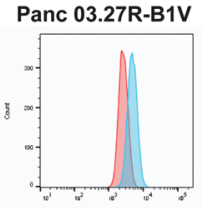

Panc 03.27R-B1Q

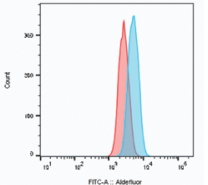

CT26.WT

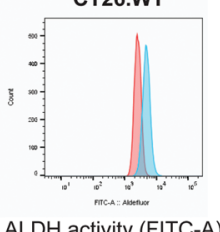

ALDH activity (FITC-A)

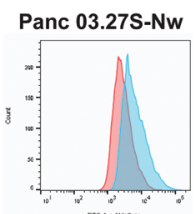

Panc 03.27S-Nt

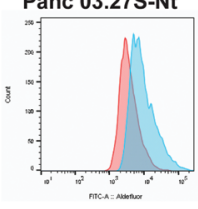

- Test sample (- DEAB)

- Control (+ DEAB)
Fig. 1 ALDH activity in 8 different cancer cell lines measured by the ALDEFLUOR assay. Representative flow results from $\geq 3$ independent experiments of both human (HT-29 and 5-FU-sensitive ( $\mathrm{Nt}$ and $\mathrm{Nw}$ ) and resistant (B1L, B1Q and B1V) sub-clones of Panc 03.27) and murine (4T1, CT26.WT) cancer cell lines.

lack of information regarding the PDT-effect on ALDH ${ }^{\text {bright }}$ versus $\mathrm{ALDH}^{\mathrm{dim}}$ cancer cells in the literature, we wanted to explore the cytotoxic effect of TPCS $_{2 \mathrm{a}}$-PDT with regard to ALDH activity within the same cell line to exclude inter-cell line genetic/proteomic variations.

Of all cell line tested, the HT-29 cell line exhibited the highest heterogeneous mixture of ALDH activity, where the median fluorescence intensity in cells incubated with BAA was more than 5 -fold higher than the DEAB control (Fig. 1). Thus, HT-29 was selected for fluorescence activated cell sorting (FACS) and subsequent evaluation of responses to chemo-, radio-, and photodynamic therapy (PDT). By means of the ALDEFLUOR assay, HT-29 cells were FACSorted into three populations: (1) Cells that exhibited very high fluorescence intensity (near $10 \%$ of the cells gated with the highest $\mathrm{BAA}^{-}$ signals), indicating high ALDH activity, were designated $\mathrm{ALDH}^{\text {bright }}$ (Fig. 2). (2) Correspondingly, cells that displayed
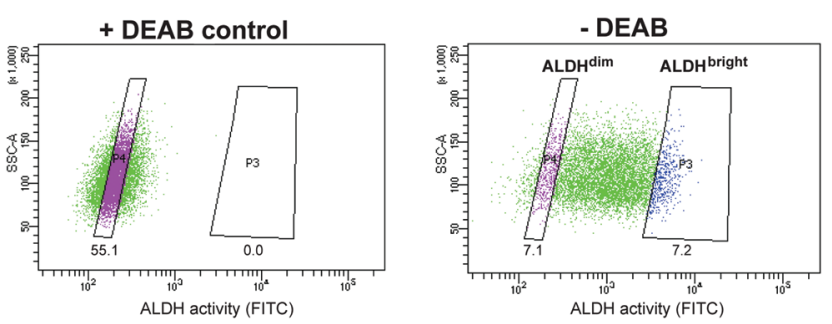

Fig. 2 Heterogeneous ALDH activity in HT-29 colon cancer cells stained with ALDEFLUOR. Representative dot-plot of live and single cells showing sorting gate for $\mathrm{ALDH}^{\mathrm{dim}}$ and $\mathrm{ALDH} \mathrm{H}^{\text {bright }}$. A control containing $D E A B$, an ALDH inhibitor, was used to set the gates. The number under each gate indicate percentage of parent population. The figure is representative of at least three independent experiments. very low fluorescence intensity (near $10 \%$ of the cells gated with the lowest $\mathrm{BAA}^{-}$signals) were defined as $\mathrm{ALDH}^{\mathrm{dim}}$. (3) Finally, unsorted cells were included to represent the bulk population. In all experiments, cells were sorted directly onto 96-well- or 6-well plates (Nunc, Thermo Fisher Scientific, Waltham, MA, USA) containing sterile filtered $(0.22 \mu \mathrm{m})$ conditioned medium mixed with fresh McCoy's 5a medium (1:1). The culture medium was supplemented with $10 \%$ fetal bovine serum, $100 \mathrm{IU} \mathrm{ml}^{-1}$ penicillin and $100 \mu \mathrm{g} \mathrm{ml}^{-1}$ streptomycin (Sigma-Aldrich). The sorted cells were allowed to attach overnight and subjected to treatment as indicated.

High ALDH activity has been associated with chemoresistance in different cancer types. ${ }^{10-13}$ We assessed the chemotherapy response of FACSorted HT-29 cells to increasing concentrations of 5-FU or oxaliplatin (both from Sigma-Aldrich) (Fig. 3A and B).

Chemotherapy-induced cytotoxic responses were measured using the MTT viability assay $\left(0.25 \mathrm{mg} \mathrm{ml}^{-1}, 4\right.$ hours incubation). Surprisingly, the cell viability was found to be similar in all FACSorted populations at all concentrations tested which indicate that ALDH activity does not significantly affect 5-FU and oxaliplatin sensitivity in the HT-29 cell line. Our results are in contrast with Kozovska et al. that reported inhibition of

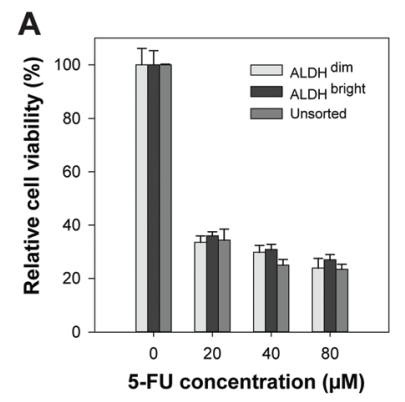

C

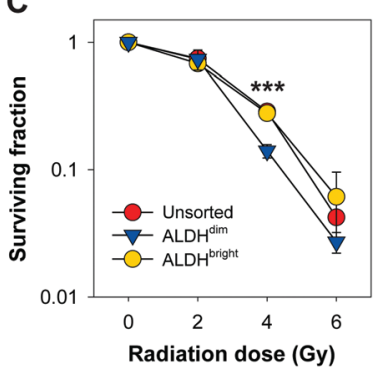

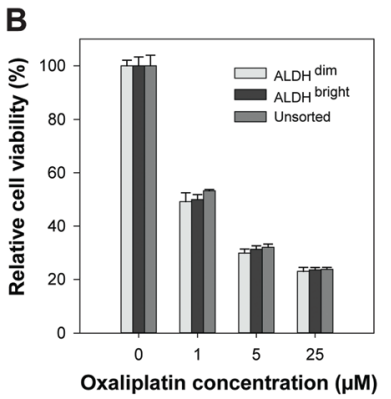

D

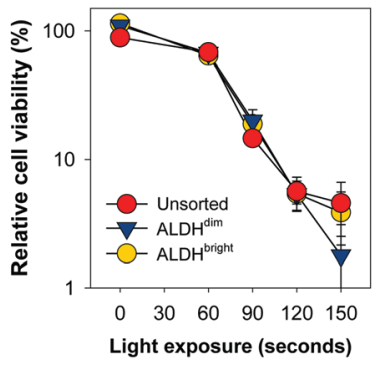

Fig. 3 Treatment sensitivity of $A L D H^{\text {dim }}, A A_{D D}{ }^{\text {bright }}$ and unsorted cells after chemotherapy, ionizing radiation or $\mathrm{TPCS}_{2 \mathrm{a}}-\mathrm{PDT}$. Relative cell viability after 72 hours incubation with (A) 5-FU and (B) oxaliplatin, measured by MTT. Representative results of three independent experiments (mean \pm S.D.). (C) Surviving fraction (SF) of HT-29 cells after increasing radiation dose, measured with clonogenic assay. Up to 14 days post-irradiation, the colonies were fixed and stained. To determine $\mathrm{SF}$, count were normalized using plating efficiency of corresponding control. Mean of three independent experiments \pm S.E. ${ }^{* * *}=p<0.001$, two-tailed $p$-value (Student's $t$-test). (D) Relative cell viability measured 72 hours post-TPCS ${ }_{2 a}$ PDT, measured with MTT assay. 60 seconds light exposure $=0.58 \mathrm{~J} \mathrm{~cm}^{-2}, \mathrm{MTT}$ data are normalized to untreated controls. Representative results of three independent experiments (mean \pm S.D.). 
ALDH using DEAB in combination with 5-FU or cisplatin significantly reduced cell viability in HT-29 cells. ${ }^{14}$ On the other hand, Prasmickaite et al. demonstrated similar sensitivity of the anti-melanoma drug dacarbazine in ALDEFLUOR-sorted cells isolated from malignant melanoma patients which indicate that ALDH alone might not be sufficient to select for chemoresistant malignant melanoma cells. ${ }^{15}$ In 5-FU- and oxaliplatin-resistant HT-29 cells, a 16-to-30 fold enrichment of the cancer stem cell marker CD133 was observed which may indicate that CD133 alone or in combination with ALDEFLUOR may be more suitable to select for resistant HT-29 cells. $^{16}$ Moreover, CD133 + cells were found to be highly resistant to 5 -FU and oxaliplatin in human colon cancer cells derived from patients. ${ }^{17}$ Data from our lab ${ }^{18}$ indicate that HT-29 exhibit high CD133 expression. As we did not include CD133 expression as a parameter for gating in our FACS, we cannot exclude that sorting based on a combination between ALDH and CD133 would have resulted in isolation of a chemo-resistant population. Therefore, the HT-29 cytotoxicity data obtained after 5-FU or oxaliplatin chemotherapy and the ALDEFLUOR assay results showing reduced ALDH activity in the 5-FU-resistant Panc 03.27 cell lines (Fig. 1) suggests that resistance to 5-FU may not be directly linked to ALDHs. ${ }^{9}$ As this is in conflict with existing literature, we suggest that more experimental research on the role of ALDH in response to 5-FU treatment is important, e.g. including ALDH knock-out models and evaluations in other cancer cell lines with heterogeneous mixture of ALDH activity.

Clonogenic assay was used to determine cell survival/death after ionizing radiation treatment of $\mathrm{ALDH}^{\text {bright }}, \mathrm{ALDH}^{\mathrm{dim}}$ and unsorted HT-29 cells in 6-well culture plates (Nunc). The cells were treated with a single fraction irradiation up to 6 Gy $(160$ kV, 6.3 mA, X-ray generator, Faxitron CP160, Tuscon, AZ, USA). When sufficiently large colonies in control plates were formed (10-14 day post-treatment), colonies were ethanol fixed, methylene blue stained and counted manually. A colony was defined to consist of at least 50 cells. ${ }^{19}$ Interestingly, based on three independent biological replicates, a slightly higher plating efficiency of $\mathrm{ALDH}^{\text {bright }}(53.3 \pm 2.5 \%)$ was observed compared to $\mathrm{ALDH}^{\mathrm{dim}}(43.0 \pm 5.3 \%$, not significant, $p=0.152)$. The plating efficiency of ALDH ${ }^{\mathrm{dim}}$ cells was also slightly lower compared to unsorted cells $(49.3 \pm 5.3 \%$, not significant, $p=$ 0.404). ALDH ${ }^{\text {bright }}$ and unsorted HT-29 cells tended towards a higher ionizing radiation resistance than $\mathrm{ALDH}^{\mathrm{dim}}$ cells but only showed a significant difference after irradiation with 4 Gy (Fig. 3C). The surviving fraction (SF) of $\mathrm{ALDH}^{\mathrm{dim}}$ cells was significantly lower ( 2-fold) at 4 Gy (SF: $14.1 \pm 0.98 \%, p<0.001$ ) compared to $\mathrm{ALDH}^{\text {bright }}(\mathrm{SF}: 27.9 \pm 1.2 \%)$ and unsorted cells (SF: $28.7 \pm 4.1 \%, p<0.001$ ). This observation is in agreement with existing studies which reported radioresistance in cells with high ALDH activity as well as in CSCs selected using other markers. ${ }^{20,21}$

PDT is based on the use of a light sensitive drug (photosensitizer) that is nontoxic in the dark and which accumulates in tumour tissues. Light exposure of the tumour tissue results in excitation of the photosensitizer leading to energy transfer from the photosensitizer to molecular oxygen $\left(\mathrm{O}_{2}\right)$ or to other cellular components, resulting in generation of cytotoxic concentration of reactive oxygen species (ROS), of which singlet oxygen $\left({ }^{1} \mathrm{O}_{2}\right)$ is the most abundant. PDT-induced ROS-generation results in peroxidation of vital cellular components and initiation of cell death mechanisms such as apoptosis, necrosis or autophagy. ${ }^{22}$

In this work, we used the clinical relevant PCI photosensitizer TPCS $_{2 \mathrm{a}}$ (fimaporfin ${ }^{23}$ ) to compare PDT efficacy in HT-29 colorectal adenocarcinoma cells with either very high or very low ALDH activity. Cells were incubated with $0.4 \mu \mathrm{g} \mathrm{ml} \mathrm{m}^{-1}$ TPCS $_{2 \mathrm{a}}$ (PCI Biotech AS) for 18 hours, washed twice with PBS and chased for 4 hours in drug-free medium to remove plasma membrane-bound TPCS $_{2 \mathrm{a}}$ to mimic a PCI protocol. The cells were subjected to broadband blue light irradiation $\left(\lambda_{\max }=\right.$ $435 \mathrm{~nm}$ ) with an output of $9.6 \mathrm{~mW} \mathrm{~cm}^{-2}$ (LumiSource, PCI Biotech AS). Cell viability was evaluated 72 hours post-light exposure by using the MTT assay, which is widely accepted in the field of PDT and has been used for 30 years to assess cell viability. ${ }^{24}$ Furthermore, we have also shown that there is a good consistency between this assay and the clonogenic cell assay. ${ }^{25,26}$ Of high interest, no statistical significant differences ( $p>0.1$ at all light exposure times) in cell viability was found between $\mathrm{ALDH}^{\mathrm{dim}}$ and ALDH ${ }^{\text {bright }}$ cells treated with $\mathrm{TPCS}_{2 \mathrm{a}}$ PDT (Fig. 3D). Of relevance, we previously demonstrated that $\mathrm{TPCS}_{2 \mathrm{a}}$ is not a substrate for the CSC markers ABCG2 and ABCB1 (P-gp) transporter ${ }^{26-28}$ which may explain why no difference in $\mathrm{TPCS}_{2 \mathrm{a}}$ sensitivity was observed in ALDH-sorted cells. As this study is on the importance of ALDH activity and its influence on TPCS $_{2 \mathrm{a}}$-PDT efficacy in only the HT-29 cell line, this should be verified in other cell lines in future studies. In addition, further investigation to establish the role of ALDH activity in PDT using other photosensitizers is warranted. In conclusion, we show that $\mathrm{ALDH}^{\mathrm{dim}}$ cells are more sensitive to ionizing radiation at 4 Gy compared to bulk and $\mathrm{ALDH}^{\text {bright }}$ populations, which is in line with the literature. However, we report that TPCS $_{2 \mathrm{a}}$-PDT is equally efficient in both $\mathrm{ALDH}^{\text {bright }}$ and $\mathrm{ALDH}^{\mathrm{dim}} \mathrm{HT}-29$ cancer cell populations. Our data further strengthen the use of TPCS $_{2 \mathrm{a}}$-based PCI of CSC-targeting therapeutics.

\section{Conflicts of interest}

There are no conflicts of interest to declare.

\section{Acknowledgements}

The present work was financially supported by South-Eastern Norway Health Authority (funding number: 2017068 (P. K. S.) and 2016023 (J. J. W. W.)) and The Norwegian Radium Hospital Research Foundation (funding number: FU0803 (P. K. S.). We would like to thank Dr. Kaja Lund and Professor Stefan Krauss (University of Oslo/Oslo University Hospital) for kindly providing us the Panc 03.27 monoclonal cell lines. We 
also thank Idun Dale Rein and Heidi Ødegaard Notø at the Flow Cytometry Core Facility (Institute for Cancer Research, Oslo University Hospital) for help with cell sorting and technical expertise.

\section{References}

1 S. S. Dinavahi, C. G. Bazewicz, R. Gowda and G. P. Robertson, Aldehyde Dehydrogenase Inhibitors for Cancer Therapeutics, Trends Pharmacol. Sci., 2019, 40(10), 774-789.

2 C. Ginestier, M. H. Hur, E. Charafe-Jauffret, F. Monville, J. Dutcher, M. Brown, et al., ALDH1 is a marker of normal and malignant human mammary stem cells and a predictor of poor clinical outcome, Cell Stem Cell, 2007, 1(5), 555567.

3 E. H. Huang, M. J. Hynes, T. Zhang, C. Ginestier, G. Dontu, H. Appelman, et al., Aldehyde dehydrogenase 1 is a marker for normal and malignant human colonic stem cells (SC) and tracks SC overpopulation during colon tumorigenesis, Cancer Res., 2009, 69(8), 3382-3389.

4 E. Batlle and H. Clevers, Cancer stem cells revisited, Nat. Med., 2017, 23(10), 1124-1134.

5 A. A. Sultan, W. Jerjes, K. Berg, A. Hogset, C. A. Mosse, R. Hamoudi, et al., Disulfonated tetraphenyl chlorin (TPCS2a)-induced photochemical internalisation of bleomycin in patients with solid malignancies: a phase 1 , doseescalation, first-in-man trial, Lancet Oncol., 2016, 17(9), 1217-1229.

6 P. K. Selbo, A. Weyergang, A. Hogset, O. J. Norum, M. B. Berstad, M. Vikdal, et al., Photochemical internalization provides time- and space-controlled endolysosomal escape of therapeutic molecules, J. Controlled Release, 2010, 148(1), 2-12.

7 R. W. Storms, A. P. Trujillo, J. B. Springer, L. Shah, O. M. Colvin, S. M. Ludeman, et al., Isolation of primitive human hematopoietic progenitors on the basis of aldehyde dehydrogenase activity, Proc. Natl. Acad. Sci. U. S. A., 1999, 96(16), 9118-9123.

8 K. Lund, J. L. Dembinski, N. Solberg, A. Urbanucci, I. G. Mills and S. Krauss, Slug-dependent upregulation of L1CAM is responsible for the increased invasion potential of pancreatic cancer cells following long-term 5-FU treatment, PLoS One, 2015, 10(4), e0123684.

9 K. Lund, C. E. Olsen, J. J. W. Wong, P. A. Olsen, N. T. Solberg, A. Hogset, et al., 5-FU resistant EMT-like pancreatic cancer cells are hypersensitive to photochemical internalization of the novel endoglin-targeting immunotoxin CD105-saporin, J. Exp. Clin. Cancer Res., 2017, 36(1), 187.

10 D. Kim, B. H. Choi, I. G. Ryoo and M. K. Kwak, High NRF2 level mediates cancer stem cell-like properties of aldehyde dehydrogenase (ALDH)-high ovarian cancer cells: inhibitory role of all-trans retinoic acid in ALDH/NRF2 signaling, Cell Death Dis., 2018, 9(9), 896.
11 L. Cortes-Dericks, L. Froment, R. Boesch, R. A. Schmid and G. Karoubi, Cisplatin-resistant cells in malignant pleural mesothelioma cell lines show ALDH(high)CD44(+) phenotype and sphere-forming capacity, BMC Cancer, 2014, 14, 304.

12 G. Vassalli, Aldehyde Dehydrogenases: Not Just Markers, but Functional Regulators of Stem Cells, Stem Cells Int., 2019, 2019, 3904645.

13 Y. Touil, W. Igoudjil, M. Corvaisier, A. F. Dessein, J. Vandomme, D. Monte, et al. Colon cancer cells escape 5 FU chemotherapy-induced cell death by entering stemness and quiescence associated with the c-Yes/YAP axis, Clin. Cancer Res., 2014, 20(4), 837-846.

14 Z. Kozovska, A. Patsalias, V. Bajzik, E. Durinikova, L. Demkova, S. Jargasova, et al., ALDH1A inhibition sensitizes colon cancer cells to chemotherapy, BMC Cancer, 2018, 18(1), 656.

15 L. Prasmickaite, B. O. Engesaeter, N. Skrbo, T. Hellenes, A. Kristian, N. K. Oliver, et al., Aldehyde dehydrogenase (ALDH) activity does not select for cells with enhanced aggressive properties in malignant melanoma, PLoS One, 2010, 5(5), e10731.

16 N. A. Dallas, L. Xia, F. Fan, M. J. Gray, P. Gaur, G. van Buren 2nd, et al., Chemoresistant colorectal cancer cells, the cancer stem cell phenotype, and increased sensitivity to insulin-like growth factor-I receptor inhibition, Cancer Res., 2009, 69(5), 1951-1957.

17 M. Todaro, M. P. Alea, A. B. Di Stefano, P. Cammareri, L. Vermeulen, F. Iovino, et al., Colon cancer stem cells dictate tumor growth and resist cell death by production of interleukin-4, Cell Stem Cell, 2007, 1(4), 389-402.

18 C. E. Olsen, L. H. Cheung, A. Weyergang, K. Berg, D. A. Vallera, M. G. Rosenblum, et al., Design, Characterization, and Evaluation of scFvCD133/rGelonin: A CD133-Targeting Recombinant Immunotoxin for Use in Combination with Photochemical Internalization, J. Clin. Med., 2019, 9(1), E68.

19 N. A. Franken, H. M. Rodermond, J. Stap, J. Haveman and C. van Bree, Clonogenic assay of cells in vitro, Nat. Protoc., 2006, 1(5), 2315-2319.

20 M. Cojoc, C. Peitzsch, I. Kurth, F. Trautmann, L. A. KunzSchughart, G. D. Telegeev, et al., Aldehyde Dehydrogenase Is Regulated by beta-Catenin/TCF and Promotes Radioresistance in Prostate Cancer Progenitor Cells., Cancer Res., 2015, 75(7), 1482-1494.

21 J. Mihatsch, M. Toulany, P. M. Bareiss, S. Grimm, C. Lengerke, R. Kehlbach, et al., Selection of radioresistant tumor cells and presence of ALDH1 activity in vitro, Radiother. Oncol., 2011, 99(3), 300-306.

22 P. Agostinis, K. Berg, K. A. Cengel, T. H. Foster, A. W. Girotti, S. O. Gollnick, et al., Photodynamic therapy of cancer: an update, Ca-Cancer J. Clin., 2011, 61(4), 250281.

23 K. Berg, S. Nordstrand, P. K. Selbo, D. T. Tran, E. AngellPetersen and A. Hogset, Disulfonated tetraphenyl chlorin (TPCS2a), a novel photosensitizer developed for clinical 


\section{Communication}

utilization of photochemical internalization, Photochem. Photobiol. Sci., 2011, 10(10), 1637-1651.

24 J. L. Merlin, S. Azzi, D. Lignon, C. Ramacci, N. Zeghari and F. Guillemin, MTT assays allow quick and reliable measurement of the response of human tumour cells to photodynamic therapy, Eur. J. Cancer, 1992, 28A(8-9), 1452-1458.

25 W. L. Yip, A. Weyergang, K. Berg, H. H. Tonnesen and P. K. Selbo, Targeted delivery and enhanced cytotoxicity of cetuximab-saporin by photochemical internalization in EGFR-positive cancer cells, Mol. Pharmacol., 2007, 4(2), 241-251.

26 P. K. Selbo, A. Weyergang, A. Bonsted, S. G. Bown and K. Berg, Photochemical internalization of therapeutic macromolecular agents: a novel strategy to kill multidrugresistant cancer cells, J. Pharmacol. Exp. Ther., 2006, 319(2), 604-612.

27 P. K. Selbo, A. Weyergang, M. S. Eng, M. Bostad, G. M. Maelandsmo, A. Hogset, et al., Strongly amphiphilic photosensitizers are not substrates of the cancer stem cell marker ABCG2 and provides specific and efficient lighttriggered drug delivery of an EGFR-targeted cytotoxic drug, J. Controlled Release, 2012, 159(2), 197-203.

28 C. E. Olsen, K. Berg, P. K. Selbo and A. Weyergang, Circumvention of resistance to photodynamic therapy in doxorubicin-resistant sarcoma by photochemical internalization of gelonin, Free Radical Biol. Med., 2013, 65, 1300-1309. 